\title{
Elderly and colorectal surgery. Analysis of surgical site infections
} Silvia Poli, Luca Degrate*, Cinzia Nobili, Mattia Garancini, Francesca Bagnariol, Fabrizio Romano, Claudio Franciosi, Roberto Caprotti and Franco Uggeri

\author{
Address: Department of Surgery, San Gerardo Hospital, University of Milano-Bicocca, via Pergolesi 33, 20052 Monza, Italy \\ * Corresponding author
}

from XXI Annual Meeting of The Italian Society of Geriatric Surgery
Terni, Italy. 4-6 December 2008
Published: I April 2009
BMC Geriatrics 2009, 9(Suppl I):A65 doi: I0. I |86/ | 47 |-23 I8-9-SI-A65

This abstract is available from: http://www.biomedcentral.com/I47I-23 I8/9/SI/A65

(C) 2009 Poli et al; licensee BioMed Central Ltd.

\section{Background}

Surgical site infection (SSI) is the most common hospitalacquired infection among surgical patients. This complication prolongs hospital stay, increases morbidity and occasionally leads to mortality.

The aim of our study is to compare incidence and characteristics of SSIs between young (under 70 years) and old (over 70 years) patients undergoing surgical colorectal resection.

\section{Materials and methods}

From November 2005 to July 2008 we prospectively collected data from patients who underwent colorectal resection. The study group comprised 264 patients, divided in two groups according to age under 70 (group A) or over 70 years (group B). We analyzed comorbidities and ASA score; type of surgical procedure and diagnosis; surgical contamination wound class, incidence of SSIs and their characteristics.

\section{Results}

Group A is composed by 144 patients ( 80 male and 64 female, mean age $58.3 \pm 9.1$ years) and group B by 120 patients ( 63 male, 57 female, mean age $77.9 \pm 5.3$ years). The two groups are comparable in term of surgical procedure, diagnosis, indication for urgent operation, surgical contamination wound class and patient BMI. Group B differ from Group A for higher ASA score, greater number of comorbidities, longer hospital stay and greater number of ostomies creation $(\mathrm{p}<0.05)$.

We didn't find out any statistically significant difference between the two groups in SSI incidence $(11 \%$ in A vs $16 \%$ in $B)$, postoperative day of SSI diagnosis $(6.8 \pm 2.7$ day in A vs $7.6 \pm 3.7$ day in B), SSI depth (12 superficial and 4 deep in A vs 13 superficial and 7 deep in B) and SSI time length $(16 \pm 13$ days in $A$ vs $17 \pm 8$ days in $B)$.

There is no statistical difference between young and old patients about incidence of SSI considering colon or rectal surgery (Right colon: $5.7 \%$ in A vs $10.4 \%$ in B; Left colon: $13.1 \%$ in A vs $26.1 \%$ in B; Rectal: $16.1 \%$ in A vs $13.3 \%$ in B).

\section{Conclusion}

Our study shows that even if the two groups differs in ASA score, number of comorbidites, hospital stay and creation of ostomy, there is not a significant difference in incidence and characteristics of SSI: as literature shows, age shouldn't be considered as a risk factor for SSIs.

A thought-provoking result is that even if we consider the site of surgery (right vs left colon vs rectum), there is not a significant difference in SSI incidence. 